\title{
Entre tolérance et concurrence
}

La communauté catholique et son identité dans l'Albanie postcommuniste

Between tolerance and competition. The Catholic community and its identity in post-communist Albania

\section{Mickaël Wilmart}

\section{OpenEdition}

\section{Journals}

Édition électronique

URL : http://journals.openedition.org/balkanologie/541

DOI : 10.4000/balkanologie.541

ISSN : 1965-0582

Éditeur

Association française d'études sur les Balkans (Afebalk)

Édition imprimée

Date de publication : 1 décembre 2004

ISSN : 1279-7952

\section{Référence électronique}

Mickaël Wilmart, « Entre tolérance et concurrence », Balkanologie [En ligne], Vol. VIII, n² 2 | 2004, mis en ligne le 20 janvier 2010, consulté le 17 décembre 2020. URL : http://journals.openedition.org/ balkanologie/541 ; DOI : https://doi.org/10.4000/balkanologie.541 


\title{
ENTRE TOLÉRANCE ET CONCURRENCE. LA COMMUNAUTÉ CATHOLIQUE ET SON IDENTITÉ DANS L'ALBANIE POST-COMMUNISTE
}

\author{
Mickaël Wilmart*
}

" Notre catholicisme est peut-être le plus primitif du monde catholique ", $^{1}$ déclarait, en 1991, Simon Jubani, prêtre à Shkodër et l'un des animateurs de la renaissance du culte catholique en Albanie après les décennies d'athéisme imposées par le gouvernement communiste ${ }^{2}$. L'absence d'encadrement des fidèles par le clergé, entre 1967 et $1990^{3}$, a, en effet, plutôt favorisé la transmission de la foi dans le cercle fermé de la famille, et donc de gestes religieux non contrôlés par le clergé, même si cette transmission est elle-même à relativiser tant le risque de délation était grand jusqu'à l'intérieur même de la famille. Le catholicisme en Albanie est une religion largement minoritaire et les pratiques religieuses manquent parfois d'orthodoxie. Si on met souvent en avant le syncrétisme des musulmans envers les pratiques chrétiennes 4 , on oublie généra-

\footnotetext{
- Ingénieur d'études, École des Hautes Études en Sciences Sociales (Paris), Centre de Recherches Historiques, Centre d'Anthropologie Religieuse Européenne, wilmart@ehess.fr
}

${ }^{1}$ Papadopoulos (Constantin), " L'Église des Catacombes n, Koha e jone (Paris), 30 (10-12), oct.-déc. 1991, p. 24. Le mot " primitif n est à comprendre ici dans une perspective évolutioniste et non dans le sens d’Église primitive.

${ }^{2}$ La religion a été officiellement interdite en Albanie en 1967 et de nouveau autorisée en 1990.

${ }^{3}$ Ce sont ici les dates de l'historien qui ne prennent en compte que le statut officiel des religions. En effet, Simon Jubani parle d'une absence du clergé " pendant deux mille ans " (cité in Papadopoulos (Constantin), art.cit.), faisant sans doute allusion à la présence ottomane jusqu'en 1913, qui, pour lui, est synonyme d'une perte d'influence de l'Église catholique, ainsi qu'à la forte présence orthodoxe au sud du pays.

${ }^{4}$ Ainsi le pèlerinage en l'honneur de Saint-Antoine de Padoue qui se déroule à Laç, au nord de Tïrana, rassemble catholiques, orthodoxes et musulmans. En 2003, le sanctuaire a attiré une foule importante, certains médias allant jusqu'à parler - exagérément - d'un million de pèlerins. Voir Ndoja (Aleksandër), «1 milione besimtare tek Kisha e shën Ndout n (un million de croyants à l'église Saint-Antoine), Koha Jonë, 14 juin 2003 (Le journal Koha Jonë est cité dans cette étude à partir de sa version mise en ligne sur internet sur le site http://www.kohajone.com). 
lement de rappeler la réciprocité du phénomène. Ainsi, le tekke de Krujë, au nord-ouest du pays, est à la fois fréquenté par des bektachis ${ }^{5}$, des musulmans et des chrétiens ${ }^{6}$. En fait, les catholiques albanais sont dans une très large majorité peu au courant du dogme et leurs pratiques religieuses sont plutôt liées à une recherche de l'efficacité7. L'identité catholique ne serait donc pas ici uniquement celle d'une pratique religieuse mais d'abord celle d'un groupe communautaire minoritaire dans un pays majoritairement musulman. Les communautés religieuses en Albanie sont en effet avant tout des groupes identitaires dans lesquels l'individu s'inscrit par tradition familiale, qu'il soit ou non pratiquant ${ }^{8}$.

La construction d'une identité religieuse passe essentiellement à travers un discours qu'il s'agit de saisir pour comprendre comment elle peut émerger dans une société multiconfessionnelle dominée numériquement par la communauté musulmane. Cette identité parait d'autant plus difficile à affirmer qu'elle doit se développer dans un discours dominant qui fait de l'Albanie un exemple de tolérance et de coexistence interreligieuse.

Pour étudier cette identité,l'approche choisie est multiple tout en restant incomplète. Plus qu'une définition, il s'agit de réfléchir aux marqueurs possibles, aux thèmes développés dans les discours des entrepreneurs identitaires (clergé et intellectuels) et à leurs échos visibles, notamment dans l'art et les débats politiques relayés par la presse. Les axes de recherches ouverts ici sont évidemment loin d'être clos. L'identité de la communauté catholique albanaise se construit en effet dans une société multiconfessionnelle mais l'étude d'un seul groupe permet de poser des questions préalables à une recherche plus large et comparée.

\section{UNE MINORITÉ CONCENTRÉE AU NORD-OUEST}

Depuis 1942, il n'y a pas eu de recensement en Albanie prenant en compte les identités religieuses ou ethniques, même si le recensement de 2001 a suscité

\footnotetext{
${ }^{5}$ Les bektachis sont membres d'une confrérie mystique musulmane hétérodoxe, la Bektachiyye, qui est devenue en Albanie une communauté religieuse à part entière après l'indépendance. Fondée en 1335 par Haji Bektach en Anatolie, sa doctrine mêle soufisme et certains usages chrétiens.

${ }^{6}$ Dervishi (Zudhi), " Restoration of the Religious Denominations and of the Democratic Culture of Tolerance in the Traditional Albanian Town : the Kruja Case n, Face to Face, juin 1996.

7 Voir l'étude de la sociologue albanaise Elira Çela, basée sur des interviews réalisées à Tirana en 1992 : Çela (Elira), "Soupçons de religion dans le système social albanais ", Conscience et liberté, (46), 1993 [Actes du symposium La liberté de conscience-fondement de la paix sociale, Tirana (Albanie), 26-28 mai 1992].

${ }^{8} \mathrm{Il}$ ne faut cependant pas perdre de vue qu'il existe un phénomène de conversion depuis 1991 dont on ne traitera pas ici.
} 
de vifs débats sur cette question. Il n'y a pas de statistiques officielles organisées par l'État et les seuls chiffres dont on dispose pour une analyse de la situation démographique sont ceux donnés par l'Église catholique elle-même9 ${ }^{9}$. Ces chiffres doivent donc être considérés avec beaucoup de précaution car il s'agit d'une autoévaluation et on en ignore la méthode d'élaboration. Toutefois, ils reflètent une certaine réalité et, s'ils ne sont pas à prendre pour une description exacte, ils donnent une tendance générale dont on peut tirer des conclusions pour comprendre la situation de la minorité catholique en Albanie. Il ne faut cependant pas perdre de vue que cette description est faite ici pour un moment donné et que la géographie de la population catholique albanaise connaît une évolution due à la situation économique du pays qui pousse les habitants du nord, notamment de Shkodër, à émigrer vers les régions centrales autour de Tirana, voire à quitter l'Albanie. En 1942, les catholiques représentaient 10,3\% de la population et en 2002 , leur part serait de $13,4 \%$, ce qui, compte tenu de l'évolution démographique du pays ${ }^{10}$, paraît tout à fait plausible et rend les chiffres donnés crédibles.

Cependant, ce chiffre brut d'un pourcentage de $13,4 \%$ sur l'ensemble du territoire n'est pas représentatif de la réalité perçue sur le terrain par tous les Albanais. En effet, il existe une forte disparité régionale de cette population qui est pratiquement absente au sud et regroupée dans les régions les plus septentrionales, et plus particulièrement au nord-ouest du pays. Ainsi, 99,85\% des catholiques sont concentrés sur $45 \%$ du territoire national, c'est-à-dire les deux archidiocèses de Durrës-Tirana (18 paroisses pour $2263 \mathrm{~km}^{2}$ ) et de Shkodër (29 paroisses pour $2363 \mathrm{~km}^{2}$ ) ainsi que les quatre diocèses de Sapë (20 paroisses pour $2544 \mathrm{~km}^{2}$ ), Lezhë (15 paroisses pour $750 \mathrm{~km}^{2}$ ), Rrëshen (17 paroisses pour 3463 $\mathrm{km}^{2}$ ) et Pult (18 paroisses pour $\left.1750 \mathrm{~km}^{2}\right)$, la moitié sud du pays étant sous l'autorité de l'administration apostolique de l'Albanie méridionale ( 6 paroisses pour $16172 \mathrm{~km}^{2}$ ). Dans cette moitié nord, les catholiques représentent $27,3 \%$ de la population et $49,4 \%$ dans les cinq diocèses les plus septentrionaux. Si on exclut l'Albanie méridionale, c'est l'archidiocèse de Tirana qui a la population catholique la plus faible ( $9,66 \%$ ) alors que c'est le diocèse de Pult (le moins peuplé et le plus au nord) qui en a le pourcentage le plus élevé (74,73\%). Cependant, le calcul de la densité de la population catholique ne donne pas les mêmes résultats, puisque les diocèses sont de taille très variable. On compte ainsi dans le plus petit diocèse, celui de Lezhë avec $750 \mathrm{~km}^{2}, 113,3$ catholiques au kilomètre carré, alors que le diocèse de Pult, qui avait le pourcentage le plus fort, n'en compte que 17,5 au kilomètre carré, ce qui s'explique par un relief montagneux peu habité.

\footnotetext{
9 Ces chiffres sont disponibles sur le site internet : $\mathrm{http}: / / \mathrm{www}$. catholic-hierarchy.org qui donne des statistiques officielles pour toute la catholicité.

${ }^{10}$ Les régions du nord où se concentre la population catholique ont connu une croissance démographique plus forte que les régions méridionales (voir Clayer (Nathalie), " God in the "Land of the Mercedes". The Religious Communities in Albania since 1990 ", Österreichische Osthefte, 45 (1-2), 2003, particulièrement p. 282).
} 
Cette disparité régionale cache en réalité une autre disparité, celle de l'encadrement des fidèles. En effet, le clergé semble particulièrement mal réparti sur le territoire albanais, même si - comme nous allons le voir - cette répartition a sa logique propre. En 1991, Simon Jubani, prêtre de Shkodër, estimait à environ 500 le nombre de paroisses nécessaires à l'encadrement de l'ensemble des fidèles catholiques albanais ${ }^{11}$. Plus de dix ans après, en 2002, on ne compte toujours que 123 paroisses. Ce chiffre n'a pas changé depuis 1999. Cependant on a assisté à un redéploiement géographique des paroisses. Les diocèses où le nombre de catholiques est le plus faible ont réduit leur nombre de paroisses afin de laisser plus de moyens aux autres régions. L'archidiocèse de DurrësTirana est ainsi passé de 19 à 18 paroisses tandis que l'administration apostolique de l'Albanie méridionale en perdait huit, passant de 14 à 6 paroisses. Ce redéploiement a profité à l'archidiocèse de Shkodër qui en a gagné deux et aux diocèses de Sapë (trois paroisses en plus) et de Lezhë qui passe de 11 à 14 paroisses. Toutefois, et malgré l'existence d'un séminaire à Shkodër, le nombre de prêtres est encore trop faible pour un encadrement adéquat. Si, sur l'ensemble de l'Albanie, on compte, en 2002, 133 prêtres (séculiers et réguliers), dont 18 albanais, pour 123 paroisses, ces chiffres cachent en réalité les difficultés que connaît localement l'Église. En effet, en dehors des archidiocèses de Shkodër et Tirana et de l'Albanie méridionale, tous les autres diocèses sont confrontés à un manque de prêtres relativement important. Ainsi, dans le diocèse de Sapë, on compte neuf prêtres pour 20 paroisses, dans celui de Rrëshen cinq prêtres pour 17 paroisses et à Pult huit prêtres pour 18 paroisses. Les situations les plus extrêmes se rencontrent dans les diocèses de Sapë et de Rrëshen, où l'on ne compte respectivement qu'un prêtre pour 10 ooo catholiques et un pour 12000 , la moyenne nationale étant de un pour 3722 . Le diocèse de Lezhë, où la densité de la population catholique est la plus forte, ne compte également qu'un prêtre pour 7083 catholiques. En fait, la population la mieux encadrée est celle située dans des régions où le taux de catholiques est le plus faible. Si l'archidiocèse de Tirana est proche de la moyenne nationale avec un prêtre pour 2612 catholiques - le statut de Tirana comme capitale du pays pourrait expliquer ce chiffre-, l'Albanie du sud possède un prêtre pour 186 catholiques !

Cependant, ces chiffres, en apparence paradoxaux, sont en fait la conséquence du statut d'Église missionnaire donnée à l'Église catholique albanaise par le clergé. C'est d'ailleurs la définition donnée dès les premières lignes d'un rapport fait à Rome en 2001 par Mgr. Angelo Massafra, président de la conférence des évêques d'Albanie : "En Albanie, l'Église est une Église missionnaire au sein d'une société multireligieuse ${ }^{12}$. Cette politique de mission évangéli-

\footnotetext{
${ }^{11}$ Voir ses propos dans Papadopoulos (Constantin), art.cit., p. 24.

12 " Intervention de Mgr Angelo Massafra, O.F.M., archevêque de Shkodër et président de la Conférence des évêques d'Alabanie, à la $10^{\text {ème }}$ Assemblée générale ordinaire du Synode des évêques (3o sept. - 27 oct.
} 
satrice s'est traduite par une augmentation sensible de l'effectif du clergé catholique en Albanie. Entre 1999 et 2002, celui-ci est passé de 102 prêtres à 133 et de 387 religieux (hommes et femmes) à 539. Les efforts déployés pour ces missions ne semblent pourtant pas avoir eu l'effet escompté13. L'encadrement plus serré de la population catholique a plutôt permis de reconsidérer à la baisse les chiffres la concernant. En 1999, l'Église annonçait environ 634300 catholiques. En 2002, ils ne seraient plus qu'environ $495000^{14} \ldots$

\section{Tableau 1 : Statistiques sur la population catholique albanaise}

\begin{tabular}{|l|c|c|c|c|c|c|}
\hline $\begin{array}{l}\text { Diocèses et } \\
\text { circonscriptions } \\
\text { religieuses }\end{array}$ & $\begin{array}{l}\text { Population } \\
\text { catholique }\end{array}$ & $\begin{array}{c}\text { Part de } \\
\text { catholiques } \\
\text { dans la } \\
\text { population } \\
\text { totale }\end{array}$ & $\begin{array}{c}\text { Nombre de } \\
\text { paroisses }\end{array}$ & $\begin{array}{c}\text { Nombre } \\
\text { de prêtres } \\
\text { (séculiers } \\
\text { et } \\
\text { réguliers) }\end{array}$ & $\begin{array}{c}\text { Nombre de } \\
\text { catholiques } \\
\text { par prêtre }\end{array}$ & $\begin{array}{c}\text { Densité } \\
\text { de la } \\
\text { population } \\
\text { catholique } \\
\text { (au km }{ }^{2} \text { ) }\end{array}$ \\
\hline Shkodër & 130000 & $65 \%$ & 29 & 47 & 2765 & 55 \\
\hline Durrës-Tirana & 96645 & $9,66 \%$ & 18 & 37 & 2612 & 42,7 \\
\hline Sapë & 90000 & $45 \%$ & 20 & 9 & 10000 & 35,4 \\
\hline Lezhë & 85000 & $70,83 \%$ & 15 & 12 & 7083 & 113,3 \\
\hline Rrëshën & 60000 & $25 \%$ & 17 & 5 & 12000 & 17,3 \\
\hline Pult & 30640 & $74,73 \%$ & 18 & 8 & 3830 & 17,5 \\
\hline $\begin{array}{l}\text { Adm. Apost. } \\
\text { Alb. Méridion. }\end{array}$ & 2800 & $0,15 \%$ & 6 & 15 & 186 & 0,17 \\
\hline Total & 495085 & $13,38 \%$ & 123 & 133 & 3722 & 16,9 \\
\hline
\end{tabular}

Source : http://www.catholic-hierarchy.org

La communauté catholique est donc largement minoritaire au niveau national et reste concentrée dans certaines régions du nord-ouest, de dialecte guègue. On peut donc considérer qu'il existe un critère géographique et linguistique dans l'identité catholique albanaise, même si, dans la pratique, cela

2001), le 4 octobre 2001 n. Retranscription des résumés des interventions publiées en ligne sur le site internet http://www.vatican.va).

${ }^{13}$ L'action missionnaire proprement dite serait évidemment à étudier en détail. Il semble qu'actuellement, elle hésite entre action humanitaire et évangélisation dogmatique. Ainsi, le témoignage publié par l'Observatoire de l'Église en Détresse sur son site internet (http://www.aed-france.org/observatoire) montre bien les difficultés rencontrées au cours des missions. En 1999, le curé de Fier a mis en place des activités caritatives dont les bénéficiaires participaient dans le même temps à des réunions de pré-catéchèse. Grâce à ce système, il était parvenu à réunir un groupe de 120 personnes qui s'est réduit à seulement cinq quand il a arrêté ses distributions.

14 Ces chiffres reflètent certainement à la fois un meilleur recensement par le clergé catholique mais aussi le résultat démographique de l'émigration albanaise vers l'étranger. 
n'apparaît que peu dans les discours. L'Église catholique affirme, quant à elle, une identité missionnaire importante. Or, cette identité missionnaire peut se comprendre de deux façons : une évangélisation des populations à rechristianiser après l'époque communiste ou une évangélisation des membres des autres communautés. Dans les deux cas, l'Église entend inscrire l'identité catholique dans un rapport d'altérité entre elle et les autres groupes confessionnels présents en Albanie.

\section{TOLÉRANCE ET JALOUSIE, LES RELATIONS INTERCONFESSIONNELLES EN ALBANIE}

L'identité missionnaire de l'Église catholique s'affirme dans un contexte interconfessionnel fortement marqué par une politique et un discours officiels de tolérance. Ce discours de tolérance et de bonne entente entre les quatre communautés traditionnelles (musulmans, bektachis, catholiques et orthodoxes) apparait comme la troisième posture des dirigeants albanais tendant à atténuer les conséquences possibles, à l'intérieur de la société albanaise mais aussi (et surtout ?) aux yeux des observateurs européens, de la cohabitation entre les différents groupes religieux composant la nation albanaise. Dès la " Renaissance " albanaise au XIX ${ }^{\mathrm{e}}$ siècle, les albanistes ont cherché à atténuer ces différences. Doit-on encore une fois rappeler la célèbre phrase de Pashko Vasa, “ Ne voyez ni églises ni mosquées, la religion des Albanais, c'est l'albanité ", reprise comme leitmotiv de toutes les politiques religieuses depuis l'indépendance ? Cette atténuation des différences s'est aussi manifestée par un discours destiné à montrer que l'Islam albanais était un Islam européen ouvert sur les autres religions et dont le bektachisme serait la manifestation la plus visible ${ }^{15}$. Le régime communiste a tenté de poursuivre cette politique en la radicalisant sous couvert d'idéologie marxiste-léniniste. C'est ainsi que, dès 1967 , toutes les religions sont interdites en Albanie. Les intellectuels communistes tentent alors de montrer la faiblesse du sentiment religieux du peuple albanais en qualifiant d'opportuniste sa conversion à l'islam. Ainsi, les Albanais n'auraient jamais été un peuple très croyant. Depuis la fin de la dictature communiste, avec l'application de la liberté de conscience ${ }^{16}$, c'est désor-

\footnotetext{
15 Sur l'utilisation politique du bektachisme en Albanie, voir Clayer (Nathalie), " Le bektachisme entre construction nationale albanaise et vision européo-centrée de l'Islam n, in Boulad-Ayoub (Josiane) et Cazzaniga (Gian Mario), éds., Traces de l'autre. Mythes de l'antiquité et Peuples du Livre dans la construction des nations méditérranéennes, Pise / Paris : Editions ETS / Librairie Philosophique Vrin, 2004.
}

${ }^{16}$ On se concentrera ici sur le discours officiel de tolérance. Sur les applications constitutionnelles et institutionnelles de la liberté de conscience, on se reportera à Clayer (Nathalie), "God in the "Land of the Mercedes"... " (art.cit.), pp. 284-287 et le rapport de Lakshman-Lepain (Rajwantee), Dimitras (Panayote), 
mais la tradition de tolérance entre les religions qui est mis en avant dans le discours officiel.

De la négation du sentiment religieux sous le communisme, les dirigeants albanais, dès les premières années de la démocratie, sont passés à l'apologie du rôle des trois religions dans l'histoire albanaise. En 1992, lors d'un colloque international sur la liberté religieuse à Tirana, le président de la République, Sali Berisha, déclarait :

Au cours de son histoire, l'Albanie a connu une cohabitation pacifique empreinte de tolérance entre les membres des trois religions principales : les musulmans, les orthodoxes et les catholiques. On le constate à l'absence de guerres religieuses. De plus, les différentes communautés ont fait preuve de respect mutuel et ont même partagé les jours fériés et les fêtes religieuses. La foi religieuse a joué un rôle significatif en unifiant le peuple albanais lorsqu'il était menacé par des forces extérieures ${ }^{17}$.

Sali Berisha insiste ici sur la tolérance en Albanie comme phénomène historique, comme une attitude inscrite dans la nature même du peuple albanais. Il met en avant une cohabitation exemplaire à un moment où une guerre oppose Serbes orthodoxes et Musulmans en Bosnie-Herzégovine. La mise en avant de cette tolérance interconfessionnelle est donc à situer dans un contexte balkanique mouvementé, dans lequel l'Albanie doit apparaître comme un facteur de stabilité. Douze ans après la déclaration de S. Berisha, un autre Président, Alfred Moisiu, tient le même propos sur l'exemplarité de la coexistence pacifique des religions en Albanie. Lors d'une interview donnée à Radio Vatican le 2 décembre 2004, il annonce l'organisation d'une conférence internationale les 9 et 10 décembre de la même année, réunissant les Présidents des pays balkaniques, sur la tolérance interethnique et interreligieuse. Il déclare à cette occasion souhaiter que " cette tradition qui existe dans le peuple albanais ne soit pas seulement connue des autres mais soit aussi apprise de nous par les autres ${ }^{18}$.

Papanikolatos (Nafsika), Catholics of Albania, Center for Documentation and Information on Minorities in Europe - Southeast Europe, septembre 2000, pp. 43-46. Pour résumer, la constitution albanaise de 1998 reconnait la liberté de conscience et donne aux communautés religieuses le statut de personnes juridiques. De plus, entre 1992 et 1998, existait un secrétariat d'État de la Religion qui a été remplacé en 1998 par un Comité d'État aux Cultes qui sert de plateforme de dialogue entre l'État et les quatre communautés religieuses principales. Notons aussi que les jours fériés ont été répartis entre les quatre confessions : l'Aïd el Fitr et l'Aïd el Kebir pour les musulmans, Noël et les Pâques catholique et orthodoxe pour les chrétiens et le Nevruz pour les bektachis.

17 " Message de M. Sali Berisha, président de la République d’Albanie ", Conscience et liberté, (46), 1993, p. 36 (numéro spécial actes du symposium La liberté de conscience - fondement de la paix sociale, Tirana, 26-28 mai 1993).

18 « Intervistë e Presidentit Moisiu dhëne Radio Vatikanit» (interview du Président Moisiu donnée à Radio Vatican), 3 décembre 2004, site de la présidence albanaise (http://www.president.al, rubrique "Intervista $"$ ). 
Comme pour les politiques précédentes, les scientifiques interviennent pour renforcer le discours officiel. Ils s'attachent ainsi à expliquer cet équilibre et à rassurer quant à sa pérennité en montrant qu'il n'y a effectivement aucun risque de conflits religieux en Albanie ${ }^{19}$. Des artistes d'horizons assez divers tentent à leur manière d'exprimer cette tolérance qui caractérise la société albanaise. Ainsi, un groupe de rap, chantant en duo avec le maire de Tirana, Edi Rama, définit Tirana comme l'endroit " où se côtoient des mosquées et des églises $n^{20}$. À Tirana toujours, dans une rue proche du centre, a été peinte sur un mur une fresque représentant les toits d'une église catholique, d'une église orthodoxe et d'une mosquée collés l'un à l'autre avec en dessous le slogan "Paix, Tolérance, Entente " (Paqe, Tolerance, Mirëkuptim) $)^{21}$.

Ce discours officiel est également relayé par les autorités religieuses tout en se gardant d'accorder la responsabilité de la tolérance aux seuls dirigeants politiques. Ainsi, pour le mufti de Tirana, Haxhi Selim Stafa, ce sont " les religions [qui] prônent la tolérance $"^{22}$ tandis que pour l'archevêque de Tirana, Rrok Mirdita, " l'harmonie entre les religions en Albanie est le mérite du peuple lui-même ${ }^{23}$. Quant au président de la Conférence des évêques d'Albanie, Mgr Angelo Massafra, archevêque de Shkodër, il déclarait en 2001 à Rome que " les rapports avec les musulmans et les orthodoxes sont caractérisés par le respect, l'acceptation et la collaboration au service des grands idéaux " et que " en général, les rapports sont excellents, même si les problèmes ne manquent pas au niveau local $n^{24}$.

Ces affirmations, relativisant parfois la politique de l'État en matière de tolérance religieuse, sont en fait assez symptomatiques de la méfiance que peuvent manifester les communautés religieuses dans leur rapport à l'État. Il existe en effet des contentieux qui voient s'organiser une action collective interconfessionnelle contre cet État juridiquement héritier du régime communiste. La question de la restitution des propriétés des communautés religieuses, qui n'est toujours pas réglée depuis 1991, a conduit, en septembre 2004, les représentants des quatre confessions (musulmans, bektachis, catho-

\footnotetext{
19 Les conclusions de ces scientifiques peuvent être diffusées dans la presse quotidienne. Voir par exemple : Fuga (Artan), " A rrezikohemi nga integrizmi fetar ne Shqipëri ? " (Y-a-t-il un danger de l'intégrisme religieux en Albanie ?), Koha Jonë, 24 novembre 2003. Ce sociologue de l'université de Tirana y met en parallèle l'absence de risque d'intégrisme religieux et la mauvaise situation socio-économique du pays qui a pour conséquence le fait que les gens ont plutôt des attentes matérielles que spirituelles.
}

${ }^{20}$ Extrait de la chanson " Tirona ", enregistrée par le groupe West Side Family et Edi Rama en 2003.

${ }^{21}$ Observation de terrain, Tirana, août 2004.

${ }^{22}$ Stafa (Haxhi Selim), «Interview ", Koha Jonë, 9 décembre 2003.

${ }^{23}$ Mirdita (Mgr Rrok), “ Harmonia fetare në Shqipëri është merite e vetë popullit n (L'harmonie entre les religions en Albanie est le mérite du peuple lui-même), Koha Jonë, 27 janvier 2004.

24 Synodus Episcoporum, (op. cit.). 
liques et orthodoxes) à se réunir pour envoyer une pétition commune au Président, au Premier ministre et au Parlement pour réclamer l'accélération de cette restitution ${ }^{25}$. Cette pétition était essentiellement motivée par les problèmes rencontrés par la communauté orthodoxe qui recherchait des locaux pour fonder une institution culturelle à Përmet. La question des propriétés touche également les lieux de culte, ceux-ci pouvant aussi être des monuments nationaux. Ainsi, le 8 janvier 1996, le Conseil des ministres a interdit les cérémonies religieuses dans une église de Shkodër certains jours de la semaine, parce que celle-ci était classée monument historique. Certains cas semblent particulièrement insolubles. Le monument abritant la " tombe " du héros national Skanderbeg à Lezhë est ainsi réclamé à la fois par les musulmans (puisqu'il est construit sur une mosquée détruite en 1967), les catholiques (il s'agirait de l'ancien emplacement de la cathédrale de Lezhë) et l'État qui le considère comme un monument national ${ }^{26}$. Les communautés n'hésitent pas non plus à brandir ensemble le spectre d'un retour à la persécution religieuse, quand l'État s'oppose à leurs prétentions. En septembre 2004, la destruction à l'explosif par la police d'une croix orthodoxe de construction jugée illégale, près de Butrint, a déclenché les réactions des clergés orthodoxe, catholique et musulman qui y ont vu les limites de la tolérance religieuse de l'État ${ }^{27}$.

Les communautés religieuses veillent également de très près à la neutralité de l'État, celui-ci ne disposant finalement que d'une marge de manœuvre très étroite pour éviter de déclencher des mécontentements quant à la sincérité de son impartialité. Si le gouvernement de Sali Berisha avait bien tenté d'équilibrer la répartition des pouvoirs étatiques entre les membres des différentes religions ${ }^{28}$, le dernier gouvernement s'est, lui, vu montrer du doigt plusieurs fois pour son attitude jugée plus favorable aux orthodoxes. Le quotidien Koha Jonë, dont le propriétaire est en fait le président du Parti démocratique chrétien (Partia DemoKristiane, PDK), d'opinion catholique, a ainsi accusé le Président Alfred Moisiu d'afficher, derrière son discours de tolérance, une préférence religieuse. On lui reproche la nomination, le 25 no-

\footnotetext{
25 " 4 komunitetet, peticion Moisiut dhe Nanos n (Les quatre communautés, pétition à Moisiu et Nano), Koha Jonë, 29 décembre 2004 .

26 Sur la question de ces propriétés, voir Lakshman-Lepain (Rajwantee), Dimitras (Panayote), Papanikolatos (Nafsika), op.cit., pp. 42-43.

27 “ Pesha e kryqit në tolerance fetare n (Le poids de la croix sur la tolérance religieuse), Koha Jonë, 15 septembre 2004 .

${ }^{28}$ Le Président Sali Berisha est musulman, le Premier ministre Aleksandër Meksi, orthodoxe et le président du Parlement, Pjetër Arboni, catholique. Cependant, le gouvernement Berisha avait plutôt favorisé par son action la communauté musulmane (voir Lakshman-Lepain (Rajwantee), " Albanie : les enjeux de la réislamisation ", in Bougarel (Xavier), Clayer (Nathalie), éds., Le nouvel Islam balkanique, Paris : Maisonneuve et Larose, 2001, pp. 133-176).
} 
vembre 2004, de quatre hauts fonctionnaires orthodoxes qui viennent s'ajouter à une liste, dressée par le journal, d'hommes politiques orthodoxes exerçant de hautes responsabilités dans l'État ${ }^{29}$. Si le journal se fait ici l'écho de l'opinion du Parti démocratique chrétien (PDK), dont le président Nikollë Lesi est intervenu au Parlement sur le sujet, cette réaction exprime aussi le climat de jalousie interconfessionnelle qui existe en Albanie et que cache, voire accentue, le discours officiel de tolérance. En effet, pour rendre concrète cette politique, l'État et ses représentants s'efforcent de maintenir un équilibre parfois impossible. Lors des fêtes de Noël 2004, le journal Koha Jonë a ainsi reproché au Président Moisiu et au Premier ministre Fatos Nano, tous les deux de confession orthodoxe, d'avoir assisté à la messe de Noël dans une église orthodoxe et d'avoir ainsi délaissé les catholiques ${ }^{3 \circ}$. Cette fois-ci, la position du PDK rejoint celle du clergé catholique qui a officiellement protesté par l'intermédiaire de l'archevêque de Tirana, Rrok Mirdita. On peut alors se demander si l'effort fait par l'État ne risque pas de conduire à une surenchère permanente. L'ouverture de l'université catholique de Tirana, en janvier 2005, a été ainsi immédiatement suivie par l'annonce d'un projet d'université musulmane par le Premier ministre Fatos $\mathrm{Nano}^{31}$.

Cette exigence surveillée de la neutralité étatique est aussi le révélateur de conflits qui viennent parfois troubler la bonne entente entre les communautés. Comme le faisait remarquer Angelo Massafra en 2001, " les problèmes locaux ne manquent pas ". Or, ces problèmes locaux sont en fait le résultat de l'activité missionnaire et prosélyte des clergés. On peut se demander dans quelle mesure le discours de tolérance a posé dans les esprits une sorte de statu quo quant à l'extension de l'influence des différentes confessions. Il y aurait des territoires réservés dans lesquelles toute action des autres communautés serait malvenue. Ainsi, l'arrivée, en 1993, de deux religieuses missionnaires catholiques dans le village orthodoxe de Gjinar, près d'Elbasan, a été très mal vécue par la communauté orthodoxe, certains allant jusqu'à comparer l'arrivée de ces deux Italiennes à "l'invasion turque ". Seul le travail caritatif des deux missionnaires a permis leur intégration dans la société villageoise ${ }^{32}$. Toute action malveillante contre des symboles religieux génère également des soupçons, la communauté lésée accusant systématiquement une autre commu-

\footnotetext{
29 " Moisiu, drekrete me preference fetare " (Moisiu, décrets avec une préférence religieuse), Koha Jonë, 26 octobre 2004 .

30 " Kreret e Shtetit, braktisin Katoliket n (Le sommet de l'État abandonne les catholiques), Koha Jonë, 26 décembre 2004. Cette réaction est particulièrement révélatrice de la place de la religion comme enjeu public en Albanie, le fait d'assister à la messe de Noël ne pouvant être considéré comme un geste privé.

${ }^{31}$ Shekulli, 22 janvier 2005.

32 " Një mision katolik në Gjinarin ortodoks " (Une mission catholique dans le [village] orthodoxe de Gjinar), Koha Jonë, $1^{\mathrm{er}}$ février 2003.
} 
nauté d'être responsable de l'acte malveillant. En janvier 2005, une polémique a ainsi éclaté après la destruction nocturne d'une croix orthodoxe de dix mètres de haut érigée au bord de la route nationale entre Tirana et Elbasan, près du village de Fikas33. L'érection de cette croix (en mai 2004) avait été auparavant contestée par la communauté musulmane d'Elbasan qui y voyait une "provocation ouverte " de la part des orthodoxes dans une région majoritairement musulmane et qui réclamait par conséquent l'application plus stricte de la loi interdisant les symboles religieux au bord des routes nationales. Sur ce dernier point, elle avait reçu le soutien de la préfecture d'Elbasan qui avait donné l'ordre de déplacer ladite croix en septembre 2004. Cependant, les orthodoxes n'ont pas accepté cette décision et ont immédiatement désigné la communauté musulmane comme coupable de l'abattage de leur croix, alors même que le mufti d'Elbasan avait condamné cet acte. Loin de calmer les esprits, cette action de vandalisme a provoqué une surenchère dans les tensions interconfessionnelles. Après avoir redressé la croix et refusé une nouvelle fois de la déplacer, la communauté orthodoxe a décidé d'instaurer une garde jour et nuit pour la protéger d'une éventuelle nouvelle attaque. Déjà en janvier 2003 , après le saccage d'une église orthodoxe à Derviçan, près de Gjirokastër, où l'autel et les objets de culte avaient été incendiés, l'Église orthodoxe d'Albanie avait désigné la communauté musulmane comme coupable 34 .

S'il n'existe pas véritablement de conflits religieux en Albanie, le discours d'une tolérance exemplaire n'est cependant pas le reflet de la réalité. Des tensions existent, visibles à la fois par une surenchère dans l'attente de la neutralité de l'État et par des actions locales qui restent symboliques. Toutefois, on peut se demander comment une Église qui se dit missionnaire peut évoluer sans heurts dans une société multiconfessionnelle dont certains dirigeants semblent revendiquer l'immuabilité de la répartition religieuse. On peut aussi se demander si le fait que les missionnaires catholiques soient dans leur quasitotalité étrangers explique les tentatives d'évangélisation dans des régions où les catholiques sont pratiquement absents et où leur action est perçue négativement au premier abord. Enfin, on doit s'interroger sur ce discours officiel de tolérance. En effet, l'effacement des conflits est aussi un effacement des différences par lequel on entend assurer l'unité de la nation. Or, pour une religion minoritaire, les seules pratiques rituelles ne suffisent pas à affirmer une iden-

\footnotetext{
33 Voir les journaux Shekulli des 12 et 13 janvier 2005, Gazeta Shqiptare des 13 et 14 janvier 2005 ainsi que Panorama du 14 janvier 2005. Comme pour Koha Jonë, nous avons utilisé les versions en ligne de ces journaux sur les sites internet http://www.shekulli.com.al, http://www.balkanweb.com/gazeta/gazeta.htm et http://www.panorama.com.al/.

34 Ces soupçons sont sans doute d'autant plus forts qu'en 1996, un groupe de jeunes musulmans dirigé par une organisation islamique avait vandalisé des fresques dans une église orthodoxe de Voskopojë (Clayer (Nathalie), "God in the "Land of the Mercedes"... " (art. cit.), p. 293, note 53).
} 
tité. Cette identité doit aussi passer par un discours qui définit la place de la communauté dans la nation sans remettre en cause celle-ci.

\section{LE DISCOURS IDENTITAIRE CATHOLIQUE ENTRE MARTYRE ET HISTOIRE NATIONALE}

Minoritaire, la communauté catholique affiche cependant une identité forte à vocation universelle. Si on pouvait penser que le discours identitaire des catholiques albanais se construirait uniquement sur le rejet de l'altérité, et particulièrement de l'altérité musulmane, le contexte de tolérance officielle l'a obligé, dans un premier temps, à construire une affirmation plus ouverte sur le reste de la société albanaise, une affirmation identitaire qui peut permettre à tous les Albanais de se retrouver dans cette Église. Deux axes sont alors privilégiés : d'une part, le martyre de l'Église albanaise pendant la période communiste qui, par extension, est l'époque du martyre du peuple albanais en son entier ${ }^{35}$; d'autre part, l'inscription de la communauté catholique dans l'histoire albanaise sur la longue durée, par la mise en avant de figures nationales catholiques.

On ne peut nier l'existence d'un sentiment de rejet vis-à-vis de la religion musulmane dans certains milieux en Albanie ${ }^{36}$. Ce discours de rejet est médiatisé avant tout par l'élite intellectuelle, mais il faut noter que ses propagateurs ne sont pas forcément chrétiens. L'exemple de l'écrivain Ismail Kadare, d'origine musulmane, est le plus connu ${ }^{37}$, mais d'autres intellectuels ont également pris position contre l'islam. Ainsi, en 2000, Edmond Tupja explique : " Nous sommes restés en marge de la civilisation chrétienne à cause de l'islam qui a empêché tout progrès chez nous ${ }^{38}$. Ce rejet intellectuel n'est cependant

35 Le sentiment de peuple martyr, victime de la dictature communiste, est largement mis en exergue au Musée d'histoire nationale de Tirana où une grande salle est consacrée au " génocide n (c'est le mot employé) dont ont été victimes les Albanais entre 1945 et 1990.

${ }^{36}$ Clayer (Nathalie), " Être Albanais dans les Balkans des années 1990 ", in Yérasimos (Stéphane), éd., Le retour des Balkans 1991-2001, Paris : Autrement, 2002, particulièrement pp. 113-114.

37 Voir Kadaré (Ismail), Printemps albanais. Chronique, lettres, réflexions, Paris : Fayard, 1991, pp. 50-51 où il souhaite le retour des Albanais au christianisme. Dans Temps barbares. De l'Albanie au Kosovo. Entretiens avec Denis Fernandez-Récatala, Paris : L'Archipel, 1999, pp. 203-204, Ismail Kadare accuse le régime d'Enver Hoxha d'avoir " accentué une prétendue singularité - celle d'une majorité musulmane ", cette majorité étant " ce que les communistes affirmaient en trafiquant les chiffres ", ceci dans une " recherche de l'isolement ". Il faut noter toutefois que l'écrivain a depuis rejoint le discours officiel de tolérance et d'harmonie religieuse (Kadare (Ismail), " Si ta ruajme harmonine e çmuar fetare n (Comment nous gardons la précieuse harmonie religieuse), Koha Jonë, 15 novembre 2003).

$3^{8}$ Cité dans Tufan (Mesut), "Quand l'Aigle (bicéphale) ne sait plus où donner de la tête ", in Yérasimos (Stéphane), éd., op. cit., p. 121. 
pas un rejet religieux, mais un rejet de civilisation. En effet, il oppose une société européenne chrétienne à une société musulmane qui rendrait l'occidentalisation impossible. Ce discours intellectuel n'est que le reflet d'un sentiment ancré plus profondément dans la population. Lors d'une enquête menée en 1992 par la sociologue albanaise Elira Çela, à la question de la raison de son désir de conversion, un adolescent, de parents musulmans, répond : " parce que nous vivons en Europe, où la religion dominante est le christianisme "39. Mais, au-delà de ce qu'on pourrait appeler un auto-rejet, le discours antimusulman peut prendre une dimension identitaire religieuse, catholique et plus largement chrétienne. Toutefois, ce rejet n'est qu'un élément parmi d'autres du discours identitaire, dont l'œuvre de l'artiste, d'origine orthodoxe mais converti aux valeurs catholiques, Maks Velo, illustre parfaitement la complexité.

La production littéraire et picturale de Maks Velo dans la période post-communiste tourne essentiellement autour de la thématique carcérale. Prisonnier de 1973 à 1986 en raison de tableaux jugés contraire au réalisme socialiste, il témoigne de son expérience vécue des camps d'internement communistes. Son témoignage est marqué d'un discours identitaire catholique très fort - sans doute d'autant plus fort que provenant d'un " converti " - dans lequel non seulement il s'oppose aux musulmans qu'il identifie aux bourreaux du peuple albanais mais dans lequel il fait aussi de son expérience carcérale une expérience de martyre chrétien. Son rejet de l'islam se manifeste dans un discours d'assimilation entre les origines musulmanes d'Enver Hoxha et la politique antireligieuse menée au nom de l'idéologie communiste. Pour lui, l'Albanie était alors un " pays dirigé diaboliquement par un musulman halluciné " : " Enver Hoxha est le plus antichrétien des dirigeants communistes de l'est. C'est le plus antieuropéen, tout cela parce qu'il vient d'une famille de hodjas. Je suis convaincu que sa lutte contre la religion vise surtout le christianisme $" 4^{\circ}$.

Dès lors, la persécution dont il a été victime, cet enfermement pour des raisons politiques, devient, sous sa plume, une épreuve du martyre qu'il présente à l'aide de métaphores christiques. Dans le camp de Spaç, assimilé au Golgotha, " les boucles de fils barbelés [sont] comme la couronne du Christ " et " les gale-

39 Çela (Elira), art. cit., p. 95.

$4^{\circ}$ Velo (Maks), Le commerce des jours. Nouvelles albanaises, Paris : Lampsaques, 1998, p. 128. Le parallèle musulmans/communistes albanais est repris dans un essai de Maks Velo sur la dictature communiste : "En Albanie, on appliquait un socialisme oriental. La dictature appliquait le code ottoman. Le dictateur Enver Hoxha était le seul dirigeant musulman parmi les premiers secrétaires des partis communistes européens. Enver Hoxha descendait d'une famille de hodjas, alors que Mehmet Shehu venait d'une famille de cheikhs " (Velo (Maks), Esse për Diktaturën Komuniste / Essay on Communist Dictatorship, Tirana : Shtëpia Botuese 55, 2003, p. 38). Il faut noter que seules les deux premières phrases du passage cité ont été traduites dans la version anglaise, la suite disparaissant du texte (Ibid., p. 153). Concernant les victimes religieuses, il clarifie son propos dans le même essai en affirmant qu'Enver Hoxha " a persécuté, emprisonné et fusillé principalement les prêtres catholiques n (ibid., p. 68). 
ries dans les montagnes comme les trous dans les mains du Christ " $4^{11}$. On retrouve cette souffrance comparée à celle du Christ dans plusieurs de ses dessins. Le sort du prisonnier dans les mines autour de Spaç s'assimile au calvaire du Christ (Krishti i galërisë [Le Christ de la galerie] ${ }^{42}$ ), le chariot qu'il pousse dans les pentes des galeries se transforme en croix, rappelant encore une fois la montée au Golgotha (Kryqi i galërisë [La croix de la galerie]43). Le prisonnier est aussi représenté comme portant la croix (Duke mbajtur kryqin [En tenant la croix] ${ }^{44}$ ) et même comme un crucifié (Kryqëzimi [Crucifixion]45).

Le martyre est en fait une des composantes les plus importantes de l'identité catholique albanaise, telle qu'elle est définie par l'Église ${ }^{46}$. Ainsi, en 2001, Angelo Massafra, archevêque de Shkodër, après avoir défini l'Église albanaise comme missionnaire devant l'Assemblée générale ordinaire du Synode des évêques réunie à Rome, poursuit sa définition en déclarant que "l'Église qui est en Albanie est une Église martyre, qui ressuscite, purifiée par la croix de la persécution " 47 . Cette mise en avant de la persécution comme facteur d'identité est largement reprise par le clergé catholique extérieur intervenant dans les affaires albanaises. Dans une homélie prononcée dans la cathédrale de Shkodër le 10 novembre 2002 au cours d'une cérémonie marquant le début du procès en béatification de 40 martyrs albanais, le cardinal Crescenzio Sepe reprend le même discours en faisant du martyre le terreau de la foi catholique en Albanie : "Si je regarde vos rues, je vois qu'elles sont partout baignées du sang des martyrs et pavées du témoignage de nombreux confesseurs du Christ. (...) C'est précisément à l'occasion de sa persécution que l'Église qui est en Albanie a rencontré le Christ et qu'elle s'est mise en route " 48 .

En fait, la thématique du martyre semble être un élément obligé du discours sur l'Église catholique albanaise, comme marqueur d'identité. Cependant, ce statut d'Église martyre ne concerne pas que la période commu-

$4^{1}$ Velo (Maks), Thesi $i$ burgut (Le sac de la prison), Tirana : Phoenix, 1996, poème " Rekiem për Spaçin " (Requiem pour Spaç), pp. 95,92 et 93.

$4^{2}$ Encre de chine publiée dans Velo (Maks), Kokëqethja, Tirana : Phoenix, 1995, p. 39.

43 Publiée dans Velo (Maks), Thesi i burgut (op. cit.), p. 34.

44 Publiée dans Velo (Maks), Kokëqethja (op. cit.), p. 21.

45 Ibid., p. 55.

$4^{46}$ Le discours sur le martyre se retrouve également chez les musulmans et les orthodoxes mais de façon moins marquée.

47 Synodus Episcoporum (op. cit.).

$4^{8}$ « Homélie du Cardinal Crescenzio Sepe, prononcée en la cathédrale de Shkodër (Albanie), le dimanche 10 novembre 2002 ", mis en ligne sur le site www.vatican.va (Curie romaine, Congrégation pour l'évangélisation des peuples). On peut ajouter à cette mention l'" Homélie du légat pontifical pour la dédicace solennelle de la nouvelle église cathédrale de Tirana (Albanie), samedi 26 janvier 2002 , sous la présidence du cardinal Angelo Sodano ", mis en ligne sur le site www.vatican.va (Curie romaine, Secrétairerie d'État). Le cardinal y parle des " nombreux martyrs qui ont sanctifié votre terre $"$. 
niste. Le 3 février 2001, le pape Jean-Paul II a rappelé aux représentants de la Conférence épiscopale de l'Albanie en visite Ad limina apostolorum que

le long chemin de l'Église catholique en Albanie a connu des moments de vitalité prometteurs et des saisons de difficulté, face aux obstacles et aux persécutions. Il suffit de rappeler la longue domination turque qui, pendant 450 ans, a mis à dure épreuve la foi des catholiques albanais et, plus près de nous, le demi-siècle de dictature communiste, qui les a obligés à vivre dans les catacombes. 49

Présence ottomane (et donc musulmane) et régime communiste sont donc ainsi mis dos à dos. Mais l'allusion à la période ottomane et à la dictature communiste renvoie aussi à deux époques où, dans l'esprit de certains, les Albanais n'étaient pas libres. Le temps du martyre des catholiques est donc aussi le temps de la souffrance du peuple albanais.

Le martyre est également présent dans les sanctuaires catholiques albanais. À Shkodër, dans la cathédrale, une chapelle abrite, en même temps que les fonts baptismaux, les photos des 40 martyrs dont le procès de béatification est en cours. Chaque baptême se fait ainsi au milieu d'images rappelant que des hommes sont morts pour leur foi, cette foi que les parents doivent inculquer à leurs enfants. Dans la même ville, dans l'église du couvent des Franciscains, une fresque représente la persécution de la foi, sous les traits de frères franciscains, par les communistes, foulard rouge au cou et entourés de démons. Là encore, le message est clair. Il s'agit de commémorer le martyre des catholiques albanais, de construire une mémoire de la persécution accessible à tous. Des discours du clergé - albanais ou étranger - aux œuvres d'art - laïques ou religieuses - en passant par l'aménagement des églises, tout est fait pour rappeler la souffrance passée. Cependant, il faut noter que certains sanctuaires récents, comme la cathédrale de Tirana, ne comportent aucune allusion à la persécution communiste. Il y aurait lieu de faire un recensement de la représentation martyrologique dans les églises albanaises pour savoir si cette absence est due au caractère récent de la construction, à sa localisation dans la capitale et donc sur une scène publique plus consensuelle ou encore au fait qu'à Tirana, les catholiques ne forment qu'une faible minorité à la mémoire déconstruite.

C'est bien en effet d'un travail de mémoire que résulte la construction d'une identité particulière aux catholiques albanais. Ce travail semble avoir été d'autant plus fort qu'il connait actuellement une dérive qui consiste à faire des seuls catholiques les victimes de la persécution communiste. On voyait déjà ce sentiment poindre chez Maks Velo, pour qui la politique antireligieuse d'un dictateur d'origine musulmane ne pouvait être qu'antichrétienne et diri- 
gée principalement contre les prêtres catholiques. Désormais, la presse à grand tirage proche des catholiques se fait aussi l'écho du statut exclusif de martyrs attribué aux catholiques albanais. Suite à la protestation adressée au Président de la République, Alfred Moisiu, au sujet de son absence aux offices des églises catholiques pour la fête de Noël 2004, le quotidien Koha Jonë déclare qu'une attention toute particulière doit être accordée aux catholiques car " tout le monde sait que le vrai massacre a été perpétré contre les représentants catholiques " 50 . Entre 1991 et 2004 , le travail de mémoire a procédé à une sélection qui semble éloigner les catholiques albanais du discours d'une communauté albanaise multiconfessionnelle, victime dans son ensemble de la politique athéiste. Si le martyre de tous les croyants albanais sous le régime communiste est devenu celui, quasi exclusif, des catholiques, c'est que la construction d'une appartenance collective par une mémoire du martyre suppose " la rencontre tragique d'une altérité ${ }^{51}$. Or, cette altérité n'existe plus qu'en opposition aux autres religions présentes en Albanie et se construit d'autant plus facilement que le groupe religieux est resté le groupe de référence identitaire à l'intérieur de la nation albanaise.

Ce travail de mémoire se double d'un travail historique. La communauté catholique, comme d'ailleurs la communauté musulmane ${ }^{52}$, justifie son identité par le passé. Elle joue même sur l'antériorité de sa présence en Albanie par rapport aux orthodoxes et aux musulmans. On peut alors s'interroger sur l'existence d'un second discours identitaire, parallèle à celui du martyre récent, basé sur une présence continue de la foi catholique en Albanie depuis les temps apostoliques et sur des figures historiques, de Saint-Paul à Mère Teresa, qui ont marqué l'histoire albanaise et qui inscrivent donc le catholicisme dans un schéma d'histoire nationale. Il s'agirait alors de ne plus baser le discours identitaire sur une opposition à l'altérité, mais au contraire de faire ressortir des figures catholiques capables de faire l'unanimité parmi les Albanais. On ne reviendra pas ici sur la figure de Skanderbeg qui, bien que surnommé " Athlète du Christ ", ne semble pas être revendiqué spécialement par la communauté catholique. La cathédrale de Tirana, consacrée en 2002, offre un meilleur exemple de cette historicité catholique. Son programme iconographique peut en effet être vu comme un résumé de l'histoire de l'Albanie catholique. Ce programme est construit autour de trois figures incontournables : Saint-Paul, Notre-Dame du Bon Conseil et Mère Teresa.

50 "Kreret e Shtetit, braktisin Katoliket „ (Le sommet de l'État abandonne les catholiques), Koha Jonë, 26 décembre 2004 .

${ }^{51}$ Albert (Jean-Pierre), "Sens et enjeux du martyre : de la religion à la politique ", in Centlivres (Pierre), éd., Saints, sainteté et martyre. La fabrication de l'exemplarité, Paris : Editions de la Maison des Sciences de l'Homme, 2001, p. 20.

${ }^{52}$ Lakshman-Lepain (Rajwantee), art. cit., p. 145. 
Saint-Paul est le patron titulaire de la cathédrale de Tirana et une chapelle lui est également dédiée. Il y est représenté debout, l'épée à la main, pointe contre terre 53 . L'apôtre a une place particulière dans l'histoire religieuse albanaise, puisqu'on sait, d'après le Nouveau Testament, qu'il a lui-même évangélisé la région alors appelée Illyrie 54 , et la tradition fait d'un de ses disciples le premier évêque de Durrës (Dyrrachium), deuxième centre urbain de l'actuel archidiocèse de Durrës-Tirana55. À l'entrée de la chapelle, comme en écho à la mission évangélique de Saint-Paul, ont été placés les fonts baptismaux. Ici, si on compare avec la cathédrale de Shkodër, la mémoire des martyrs a donc été remplacée par la mémoire des origines, une mémoire sans doute moins polémique.

La deuxième chapelle de la cathédrale est placée sous l'invocation de la Vierge. Celle-ci est représentée par une statue, mains ouvertes et tendues vers le bas, le pied droit écrasant un serpent entourant le monde et mordant dans une pomme symbole du péché originel. Cette statue mariale est prolongée - et prend son sens dans un contexte albanais - par l'iconographie des vitraux placés à droite de la chapelle. Ceux-ci racontent en effet l'épisode de la translation miraculeuse de l'icône de Notre-Dame du Bon Conseil que la légende a rapproché de la mort de Skanderbeg. Quelques semaines après la mort du héros national, les Ottomans auraient assiégé la ville de Shkodër ${ }^{56}$, au nord du pays. Pendant le siège, une image représentant la Vierge et le Christ enfant quitte l'église de Notre-Dame du Bon Conseil, portée par des anges, et migre vers l'Italie, suivie par deux pèlerins, pour s'arrêter à Genazzano, près de Rome. Cette " fuite ", à un moment où la distorsion chronologique de l'imaginaire albanais ${ }^{57}$ fait basculer Shkodër dans l'Empire ottoman, symbolise la fin de l'hé-

53 L'ensemble de vitraux le plus important de la cathédrale, au dessus de la tribune, lui est également consacré et retrace sa vie.

$54 \mathrm{Rm}$ 15, 19 : “ depuis Jérusalem et les environs jusqu'en Illyrie, j'ai porté partout l'Evangile du Christ ". Son passage serait intervenu lors de son troisième voyage qui le conduit vers la Macédoine toute proche (Ac 20, 1-3).

55 Voir une analyse des textes concernant les premiers temps chrétiens en Albanie dans Frashëri (Kristo), "Fillimet e krishterimit në viset shqiptare " (Les débuts du christianisme en pays albanais), Studime Historike, 54 (1-2), 2000. Il faut noter ici que l'appellation " archidiocèse de Durrës-Tirana ", alors que le siège se trouve à Tirana, renvoie aux origines historiques du christianisme albanais, puisque le premier diocèse fondé en Albanie serait celui du Durrës, au premier siècle après Jésus-Christ.

${ }^{56}$ Les sièges de Shkodër ont en réalité eu lieu en 1473 et 1479.

57 On trouve une trace de cette distorsion chronologique dès la moitié du XVIII'me siècle. Ainsi, en 1748, le procureur des missionnaires apostoliques, Nicolas di Antonio Cambsi, originaire de Shkodër, déclare que l'image sainte est partie " avant que la ville épuisée tombât aux mains des Turcs ". (attestation du 9 février 1748, publiée dans Dillon (Gilles F.), La Vierge Mère de Bon Conseil, Liège : Desclée / De Brouwer, 1885, p. 460). Cependant, le clergé catholique de la fin du XIX ${ }^{e}$ siècle est déjà conscient de ce problème de chronologie et Angelo Radoja, vicaire général de Shkodër, rectifie en 1878 en situant la translation miraculeuse " quelques années avant que les Turcs prissent possession de Scutari " (lettre du 17 décembre 1878, publiée dans ibid., p. 473). 
gémonie chrétienne en Albanie. Lieu de la dernière manifestation divine à Shkodër avant les siècles de présence ottomane, l'emplacement - supposé - de Notre-Dame de Shkodër et de l'église après ses reconstructions est resté un lieu de pèlerinage extrêmement fréquenté. Une dimension nationale a été donnée en 1895 à Notre-Dame du Bon Conseil, quand un concile d'évêques albanais en a fait la sainte patronne de l'Albanie. Notre-Dame du Bon Conseil est depuis le titre sous lequel elle est le plus invoquée en Albanie, comme le rappelait le cardinal Sodano lors de la consécration de la cathédrale de Tirana en 2002. Sa présence dans la cathédrale entre donc aussi dans un programme historique réunissant les figures incontournables de l'Albanie chrétienne.

La dernière figure présente dans la cathédrale est Mère Teresa, béatifiée le 19 octobre 2003 et à laquelle on a consacré un vitrail dans la chapelle SaintPaul, réunissant dans un même espace l'évangélisateur et celle qu'on considère en Albanie comme la dernière " sainte " albanaise en date. La présence de Mère Teresa dans la cathédrale catholique de Tirana est révélatrice d'une volonté de mêler identité catholique et identité nationale. En effet, si Mère Teresa est bien une religieuse catholique béatifiée par le pape, certains, en Albanie, essayent de l'ériger en figure nationale. Il suffit de rappeler la querelle entre la Macédoine et l'Albanie sur l'origine géographique de la religieuse de Calcutta pour mesurer l'attachement de l'Albanie à " sa sainte ". Le 19 octobre est d'ailleurs devenu jour férié en souvenir de sa béatification. Dès l'annonce de celle-ci, un discours s'est élaboré visant à faire de la Bienheureuse Teresa de Calcutta la " sainte Teresa des Albanais " pour reprendre le titre d'un article du journal Koha Jonë ${ }^{5}$. Dans cet article, l'auteur, Bardhyl Ukçamaj, fait entrer Mère Teresa dans le "panthéon d'or que l'Église Illyro-Albanaise a donné à l'Église catholique universelle ". Mêlant Illyrie au sens le plus large et Albanie actuelle, il crée une généalogie sacrée montrant que Mère Teresa est en fait le fruit d'une histoire catholique locale de longue durée : saint Eleuthère, évêque d'Illyrie, originaire de Rome, qui aurait siégé à Vlorë et que l'auteur confond avec son homonyme, pape de 175 à 189 ; saint Caïus, pape de 283 à 296 originaire de Dalmatie ; saint Astius, évêque de Durrës et martyr en 98 ; saint Therinus, originaire de Butrint et martyr du $\mathrm{III}^{\mathrm{e}}$ siècle ; saint Donat, originaire également de Butrint et évêque en Epire à la fin du IV ${ }^{\mathrm{e}}$ siècle ; les saints Florus et Laurus, originaires de Constantinople et martyrisés au sud de Priština ; un certain saint Dardani qui semble être né d'une confusion avec une province ecclésiastique illyrienne ; saint Jérôme ${ }^{59}$, illyrien de Dalmatie ; un saint Alban dont le lien avec l'Albanie n'est qu'étymologique ; sainte Angelina de Krujë,

${ }^{8}$ Ukçamaj (Bardhyl), « Shën Tereza e Shqiptareve " (Sainte Teresa des Albanais), Koha Jonë, 3 septembre 2003.

59 On notera l'importance de l'origine albanaise prêtẻe à Saint-Jérôme, auteur de la Vulgate qui a donné le texte biblique à toute l'Europe occidentale ! 
belle-sœur de Skanderbeg (mais reconnue sainte seulement par l'Église orthodoxe serbe). Cette généalogie sacrée, construite avec peu de rigueur, inscrit Mère Teresa parmi les saintes figures de l'Albanie, mais elle inscrit également la communauté catholique dans une histoire du pays sur la longue durée. Elle se poursuit par l'évocation de personnages ayant servi l'Église et d'origine également albanaise ou supposée telle comme Skanderbeg, Lekë Dukagjini, le pape Clément XI (dont le nom de famille est Albani) ou encore les 40 martyrs dont le procès de béatification est en cours. Comme pour justifier son propos, l'auteur de l'article poursuit en rappelant les signes envoyés par Mère Teresa au peuple albanais en 1979, lors de la remise de son prix Nobel où elle déclara penser " au peuple et à l'Église albanais " ou encore en 1988 lorsqu'elle prononça une prière et un discours en albanais pour la communauté albanaise du Bronx à New York dans l'église Zoja e Shkodrës (Notre-Dame de Shkodër, c'està-dire Notre-Dame du Bon Conseil).

Cependant, malgré ce discours, la confessionnalisation de la figure de Mère Teresa n'est pas évidente dans le paysage albanais. Outre le jour de sa béatification devenu jour férié, il faut rappeler le nombre important d'hommages rendus à la religieuse par les autorités laïques de l'État. On ne compte plus, en effet, le nombre de places ou d'écoles portant son nom, jusqu'à l'aéroport international de Rinas-Tirana. En 2003, le ministère de la Culture et la Galeria Kombëtare e Arteve (Galerie Nationale des Arts) ont organisé un concours pour la création d'une statue de Mère Teresa, gagné en 2004 par Thoma Thomai. À l'occasion de l'inauguration de la statue, le Premier ministre Fatos Nano a renouvelé les propos des discours politiques habituels qui, s'ils ne nient pas l'origine catholique de Mère Teresa, insistent surtout sur le fait qu'elle était d'abord albanaise. Ainsi, pour lui, " dans la figure de Mère Teresa s'incarnent les meilleures qualités des mères albanaises "60. L'érection de cette statue a provoqué un début de polémique sur son emplacement, jugé trop excentré et peu visible, et son programme artistique auquel on reproche une totale absence du message de Mère Teresa ${ }^{61}$. L'artiste a préféré rendre hommage à la figure albanaise plutôt qu'à la religieuse. La polémique esquissée - qui n'a pas eu de suite - est sans doute le signe d'une volonté de ne pas ôter à Mère Teresa son message religieux. Toutefois, Enver Faja, à l'origine du

\footnotetext{
60 " Discours du Premier ministre de la République d'Albanie, 9 décembre 2004 ", publié sur le site officiel du Premier ministre (http://keshilliministrave/al). Dans ce discours, Fatos Nano, comme le Président Moisiu dans son interview accordée à Radio Vatican (2 décembre 2004), répètent la " fierté n des Albanais de compter Mère Teresa parmi leurs compatriotes.
}

${ }^{61}$ Voir sur cette statue et sa polémique : Faja (Enver), « Mbi monumentin e Nënë Terezës, inauguruar një muaji pranë Universitetit të Tiranës : ky monument është një gabim " (À propos du monument de Mère Teresa, inauguré il y a un mois près de l'université de Tirana : ce monument est une erreur), Shekulli, 7 janvier 2005. 
débat, met en garde contre une trop forte identification de Mère Teresa à l'Albanie et aux seuls catholiques. Pour lui, il s'agit de ne pas oublier que le message de Mère Teresa est universel. Cette mise en garde est le symptôme d'une manifestation identitaire de plus en plus forte en Albanie, où l'on a préféré construire la mémoire de la religieuse de Calcutta uniquement comme figure nationale albanaise.

Mais la figure de Mère Teresa tend à s'inscrire dans l'affirmation identitaire des seuls catholiques albanais. Ce n'est pas pour rien que la première œuvre d'art exposée sur Mère Teresa en Albanie est un vitrail de la cathédrale catholique de Tirana. Auparavant, Maks Velo avait tenté de mettre en parallèle le discours martyrologique, à la base du discours identitaire catholique, et la figure de Mère Teresa. Dans deux poèmes publiés en 1996 dans le recueil Thesi $i$ burgut (Le sac de la prison), il laisse apparaître l'espoir que représentait Mère Teresa pour le prisonnier qu'il était ${ }^{62}$. En 2003, il a également publié un recueil de dessins consacrés à Mère Teresa ${ }^{63}$, mais dans lequel il a aussi inclus les dessins d'inspiration religieuse qui ont été analysés plus haut. En mettant côte à côte la souffrance christique du prisonnier et des portraits de Mère Teresa, il rejoint le discours de Bardhyl Ukçamaj dans Koha Jonë visant à créer une généalogie entre l'histoire catholique albanaise et elle, et donc à reconfessionnaliser sa figure. Enfin, en octobre 2004, le peintre a organisé une exposition de ses dessins sur Mère Teresa, dans laquelle il entendait faire passer le message de foi chrétienne de la religieuse ${ }^{64}$.

Depuis 1991, la communauté catholique albanaise est en reconstruction. L'affirmation de son identité, en dehors de ses pratiques cultuelles, se résume en trois points : une Église missionnaire, une Église et une communauté martyres et une communauté participant à l'histoire nationale depuis près de deux mille ans. Cette affirmation se pose parfois en rejet de l'altérité (surtout musulmane) mais celle-ci s'élabore principalement par un travail de mémoire.

\footnotetext{
${ }^{62}$ Velo (Maks), " Nënë - emri yt " (Mère, ton nom) et « Nënë Terezës n (Mère Teresa), in Thesi i burgut (op. cit.), pp. 31-32, 84.

${ }^{6}$ Velo (Maks), Albume "Nënë Tereza ", Tirana : Shtëpia Botuese 55, 2003. Une deuxième édition a été imprimée en 2004. Il faut noter que l'éditeur de Maks Velo, Fahri Balliu, directeur des éditions 55, est un converti au catholicisme ("Fahri Balliu, Katolik i ri " (Fahri Balliu, le nouveau catholique), Koha Jonë, 29 mars 2004).

64 " Një biografi grafike për shenjtpren shqiptare " (Une biographie graphique pour la sainte albanaise), Koha Jonë, 26 octobre 2004 .
} 
Le discours officiel de tolérance, s'il est le reflet d'une relative bonne entente entre les communautés, efface cependant leurs différences et cache leurs conflits qui manifestent pourtant une volonté d'affirmer une identité particulière sans jamais renier l'appartenance à la nation. Cet effacement des différences conduit sans doute à une surenchère à la fois dans l'attente de neutralité de la part de l'État, mais aussi vis-à-vis des autres confessions. Il oublie aussi une réalité de terrain dans laquelle les membres des communautés vivent dans des quartiers ou des villages différents ${ }^{65}$ (c'est le cas par exemple à Shkodër) et gardent de forts a priori sur les autres, même si les mariages interconfessionnels se multiplient depuis la chute du régime communiste.

Il ne faut cependant pas perdre de vue que la construction identitaire de la communauté catholique albanaise - comme des autres communautés - est toujours en cours. La mémoire du martyre et la question de la confessionnalisation ou déconfessionnalisation de la figure de Mère Teresa sont encore amenées à évoluer et seront déterminantes dans la définition de l'identité catholique en Albanie dans les prochaines années.

65 Ce phénomène a tendance à disparaître dans les centres économiques comme Tirana où l'évolution démographique due à l'émigration intérieure fait se côtoyer dans les nouveaux quartiers des habitants de confessions et d'horizons différents. 